\section{EVIDENCE THAT INTRAUTERINE GROWTH RESTRICTION MAY ENHANCE TYPE III COLLAGEN SYNTHESIS IN FULL-TERM PREGNANCIES}

\author{
D.D. Briana, D. Gourgiotis, M. Boutsikou,
} A. Kakaroukas, L. Stamati, A. Georgiadis, S. Baka, D. Hassiakos, A. Malamitsi-Puchner

Athens University Medical School, Athens, Greece

Background and aims: N-terminal propeptide of type-III procollagen (PIIINP) is a marker of type III collagen synthesis, reflecting overall growth and tissue maturity. We aimed to prospectively investigate circulating PIIINP concentrations in intrauterine- growth-restricted (IUGR) and appropriate-for-gestational-age (AGA) mother/ infant pairs at term.

Methods: Serum concentrations of PIIINP (a circulating marker of type III collagen synthesis) were measured by RIA in 40 mothers and their 20 asymmetric IUGR (adjusted birthweight $\leq 5^{\text {th }}$ customized centile) and 20 AGA singleton full-term fetuses and neonates on postnatal day 1 (N1) and 4 (N4). Results: Fetal, N1 and N4 concentrations were significantly higher in the IUGR group $(p=0.015$, $p=0.017$ and $p=0.003$, respectively). In both groups, maternal PIIINP concentrations were lower than fetal, N1 and N4 ones ( $p<0.001$ in each case). In a combined group, maternal PIIINP concentrations positively correlated with $\mathrm{N} 1$ and $\mathrm{N} 4$ ones $(r=0.321$, $p=0.043$ and $r=0.412, p=0.008$, respectively). The effect of gender, delivery mode and parity on PIIINP concentrations was not significant.

Conclusions: Contrary to our expectations fetal/neonatal circulating PIIINP concentrations were increased in IUGR cases as compared to AGA controls. We speculate that this fact should be attributed to the stress-related IUGR state, responsible for induction of tissue maturation. Higher fetal/neonatal PIIINP concentrations as compared to maternal ones should be related to higher collagen turnover in the former. Lastly, positive correlations of PIIINP between mother and offspring could imply a transplacental passage of the protein.

\section{5}

\section{EFFECT OF ENTERAL SUPPLEMENTATION OF NEUTRAL AND ACIDIC OLIGOSACCHARIDES ON SERUM CYTOKINE LEVELS IN PRETERM INFANTS}

E. Westerbeek, N. van Zwieteren, H. Lafeber, R. van Elburg

\section{Neonatalogy, VU University Medical Center, Amsterdam, The Netherlands}

Introduction: Preterm have an immature immune system. Aim of this study was to determine the effect of a prebiotic mixture consisting of neutral and acidic oligosaccharides ( ${ }_{S C} \mathrm{GOS} /{ }_{\mathrm{LC}} \mathrm{FOS} / \mathrm{AOS}$ ) on cytokine levels in the blood of preterm infants.

Methods: In a RCT, preterm infants (gestational age $<32$ weeks and/or birth weight $<1500 \mathrm{~g}$ ) received ${ }_{S C} \mathrm{GOS} /{ }_{L C} F O S / A O S$ or maltodextrin (placebo) between days 3 and 30 of life. Cytokine levels (IL-1 $\beta$, IL-2, IL-4, IL-6, IL-8, IL-10, IL-17, IFN- $\gamma$, TNF- $\alpha$ ) were analysed by a fluorescent bead-based multiplex immuno assay at 3 time points: before the start of the study, day 7 and day 14 .

Results: In total, 113 infants were included. Baseline patient and nutritional characteristics were not different in the ${ }_{S C}$ GOS/ ${ }_{L C} F O S / A O S(n=55)$ and the placebo group $(n=58)$. Enteral supplementation of ${ }_{S C} \mathrm{GOS} /{ }_{L C}$ FOS/AOS did not change cytokine levels. There was a trend toward lower levels of IL$1 \beta(P=0.05,95 \% \mathrm{Cl} 0.15-1.01)$ and TNF- $\alpha(P=0.14$, $95 \% \mathrm{Cl} 0.18-1.26)$ in ${ }_{S C} \mathrm{GOS} /{ }_{L C} \mathrm{FOS} / \mathrm{AOS}$ group compared with the placebo group. Adjustment for serious infectious morbidity did not change the results of the primary analysis.

Conclusions: There is a trend toward decreased levels of the pro-inflammatory cytokines IL-1 $\beta$ and TNF- $\alpha$ after enteral supplementation with a prebiotic mixture consisting of neutral and acidic oligosaccharides. Increased pro-inflammatory cytokines are associated with multi-organ failure, chronic lung disease and white matter damage and therefore we speculate that a prebiotic mixture consisting of neutral and acidic oligosaccharides may improve clinical outcome in preterm infants. 